\title{
Blow-Up Criteria for the Modified Novikov Equation
}

\author{
Caochuan $\mathrm{Ma}^{1}$ and Wujun $\mathrm{Lv}^{2}$ \\ ${ }^{1}$ School of Mathematics and Statistics, Tianshui Normal University, Tianshui 741001, China \\ ${ }^{2}$ Department of Mathematics, Zhejiang Normal University, Jinhua 321004, China
}

Correspondence should be addressed to Caochuan Ma; ccmamath@gmail.com

Received 27 March 2014; Accepted 15 April 2014; Published 30 April 2014

Academic Editor: Yonghui Xia

Copyright ( 2014 C. Ma and W. Lv. This is an open access article distributed under the Creative Commons Attribution License, which permits unrestricted use, distribution, and reproduction in any medium, provided the original work is properly cited.

We investigate the Cauchy problem for the modified Novikov equation. We establish blow-up criteria on the initial data to guarantee the corresponding solution blowing up in finite time.

\section{Introduction}

In this paper, we consider the following Cauchy problem of the modified Novikov equation:

$$
\begin{gathered}
u_{t}-u_{x x t}+(a+b) u^{2} u_{x} \\
=a u u_{x} u_{x x}+b u^{2} u_{x x x}, \quad t>0, x \in \mathbb{R}, \\
u(0, x)=u_{0}(x), \quad x \in \mathbb{R},
\end{gathered}
$$

where the coefficients $a$ and $b$ are positive constants.

In [1], Lai et al. presented the global existence of strong solutions and gave a blow-up scenario of strong solutions to the equation.

By using Green's function $G(x)=(1 / 2) e^{-x}$ for the operator $\Lambda=\left(1-\partial_{x}^{2}\right)^{1 / 2}$, (1) is equivalent to the nonlocal equation

$$
\begin{gathered}
u_{t}+b u^{2} u_{x} \\
=G *\left[-a u^{2} u_{x}+\frac{a-6 b}{2}\left(u u_{x}^{2}\right)_{x}+\frac{2 b-a}{2} u_{x}^{3}\right] \\
t>0, x \in \mathbb{R}, \\
u(0, x)=u_{0}(x), \quad x \in \mathbb{R},
\end{gathered}
$$

where notation $*$ denotes the spatial convolution.

Letting $a=3 b$ and using the scaling translation $\tilde{u}=b u$, (1) can be reformulated into the Novikov equation

$$
\tilde{u}_{t}-\tilde{u}_{x x t}+4 \widetilde{u}^{2} \tilde{u}_{x}=3 \tilde{u} \widetilde{u}_{x} \tilde{u}_{x x}+\widetilde{u}^{2} u_{x x x},
$$

which was derived by Novikov in a symmetry classification of nonlocal PDEs with quadratic or cubic nonlinearity [2]; subsequently, he found a scalar Lax pair for the Novikov equation (also see [3]) and proved that the Novikov equation is integrable. The equation has been investigated by many scholars. Hone and Wang gave a matrix Lax pair for the Novikov equation in [4] and showed how it was related by a reciprocal transformation to a negative flow in the SawadaKotera hierarchy. By using the matrix Lax pair, Hone et al. calculated the explicit formulas for multipeak on solutions of (1) in [3]. Ni and Zhou showed that the Novikov equation is well-posed in $H^{s}, s>3 / 2$ by applying Kato's semigroup theory and the Novikov equation is locally well-posed in the Besov spaces $B_{2, r}^{s}$ with the critical index $s=3 / 2$ and also considered the persistence properties of the solution. In [5], Jiang and $\mathrm{Ni}$ gave sufficient conditions on the initial data to guarantee the formulation of singularities in finite time and a global existence result was also established in [6]. It is worth pointing out recent many works have been done for the Novikov equation and the related equations, one can refer to [7-12] and the references therein.

Now, we give some elementary results and a blow-up scenario of strong solutions which will be used in this paper.

Theorem 1 (see [13]). Given $u(x, t=0)=u_{0} \in H^{s}(\mathbb{R})$ with $s>3 / 2$, then there exist a maximal $T=T\left(u_{0}\right)>0$ and $a$ unique solution $u$ to (1) such that

$$
u=u\left(\cdot, u_{0}\right) \in C\left([0, T) ; H^{s}(\mathbb{R})\right) \cap C^{1}\left([0, T) ; H^{s-1}(\mathbb{R})\right) .
$$


Moreover, the solution depends continuously on the initial data; that is, the mapping $u_{0} \rightarrow u\left(\cdot, u_{0}\right): H^{s}(\mathbb{R}) \rightarrow$ $C\left([0, T) ; H^{s}(\mathbb{R})\right) \cap C^{1}\left([0, T) ; H^{s-1}(\mathbb{R})\right)$ is continuous.

Theorem 2 (see [1]). Assume $u_{0}(x) \in H^{s}, s \geq 3 / 2$, and let $T$ be the maximal existence time of the solution $u(x, t)$ to (1) with the initial data $u_{0}(x)$. If $a>b$, then the corresponding solution blows up in finite time if and only if

$$
\lim _{t \uparrow T} \liminf _{x \in \mathbb{R}}\left(u u_{x}\right)(x, t)=-\infty .
$$

We also need to introduce the classical particle trajectory method. Suppose $u(x, t)$ is a solution of the Novikov equation; let $q(x, t)$ be the particle line evolved by the solution $u$ :

$$
\begin{gathered}
\frac{d q(x, t)}{d t}=b u^{2}(q(x, t), t), \\
q(x, t=0)=x .
\end{gathered}
$$

Then

$$
q_{x}(x, t)=\exp \left(2 \int_{0}^{t} u u_{x}(q, s) d s\right), \quad q_{x}(x, 0)=1
$$

which is always positive before the blow-up time. Therefore, the function $q(x, t)$ is an increasing diffeomorphism of the line before blow-up.

Let $y=\Lambda^{2} u=\left(1-\partial_{x}^{2}\right) u$; the following identity can be obtained:

$$
y(q(x, t), t) q_{x}^{2}(x, t)=y_{0}(x) e^{(4 b-a) \int_{0}^{t} u u_{x} d s} .
$$

In fact, direct computation yields

$$
\begin{aligned}
\frac{d}{d t}( & \left(q(q) q_{x}^{2}\right) \\
= & y_{t} q_{x}^{2}+2 y q_{x} q_{x t}+y_{x} q_{t} q_{x}^{2} \\
= & y_{t} q_{x}^{2}+4 b y u u_{x} q_{x}^{2}+b u^{2} y_{x} q_{x}^{2} \\
= & \left(u_{t}-u_{t x x}+a u u_{x}\left(u-u_{x x}\right)+b u^{2}\left(u_{x}-u_{x x x}\right)\right) q_{x}^{2} \\
& -a u u_{x} y q_{x}^{2}+4 b u u_{x} y q_{x}^{2} \\
= & (4 b-a) u u_{x} y q_{x}^{2} .
\end{aligned}
$$

Remark 3. From (8), it follows that if $y_{0}(x)=\Lambda^{2} u_{0}(x) \geq 0$ then $y(t, x)=\Lambda^{2} u(t, x) \geq 0$. Since $\Lambda^{-2} f=G * f$, for $f \in$ $L^{2}(\mathbb{R})$, therefore, we obtain $u(x) \geq 0$. If $y_{0}=\left(1-\partial_{x}^{2}\right) u_{0}(x) \leq 0$, the result is similar.

\section{Blow-Up Criteria}

In this section, we present the following blow-up criteria on the initial data to guarantee that the corresponding strong solution of (1) blowing up. Our method is partially motivated by [14].
Theorem 4. If $a>3 b$, suppose that $u_{0} \in H^{s}(\mathbb{R}), s>3 / 2$, and there exists $x_{0} \in \mathbb{R}$ such that $u_{0}\left(x_{0}\right) \geq 0$ and $y_{0}\left(x_{0}\right)=$ $\left(1-\partial_{x}^{2}\right) u_{0}\left(x_{0}\right)=0$,

$$
\begin{aligned}
& y_{0}(x) \geq 0(\not \equiv 0) \quad \text { for } x \in\left(-\infty, x_{0}\right), \\
& y_{0}(x) \leq 0(\not \equiv 0) \quad \text { for } x \in\left(x_{0}, \infty\right) .
\end{aligned}
$$

Then the corresponding solution $u(x, t)$ to the modified Novikov equation (1) with $u_{0}(x)$ as the initial datum blows up in finite time.

Proof. By the local well-posedness theorem and a density argument, it suffices to consider the case $s \geq 3$; without loss of generality, we take $s=3$ for simplicity of notation. We also assume $u_{0} \not \equiv 0$; otherwise, solutions are trivial.

Suppose that the solution exists globally. Due to (8) and the initial condition (10), we have

$$
\begin{gathered}
y\left(q\left(x_{0}, t\right), t\right)=0, \\
y(q(x, t), t) \geq 0(\not \equiv 0), \quad \text { for } x \in\left(-\infty, x_{0}\right), \\
y(q(x, t), t) \leq 0(\neq \equiv 0), \quad \text { for } x \in\left(x_{0}, \infty\right),
\end{gathered}
$$

for all $t \geq 0$. Since $u(x, t)=G * y(x, t), x \in \mathbb{R}, t>0$, we can write $u(x, t)$ and $u_{x}(x, t)$ as follows:

$$
\begin{gathered}
u(x, t)=\frac{1}{2} e^{-x} \int_{-\infty}^{x} e^{\xi} y(\xi, t) d \xi+\frac{1}{2} e^{x} \int_{x}^{\infty} e^{-\xi} y(\xi, t) d \xi \\
u_{x}(x, t)=-\frac{1}{2} e^{-x} \int_{-\infty}^{x} e^{\xi} y(\xi, t) d \xi+\frac{1}{2} e^{x} \int_{x}^{\infty} e^{-\xi} y(\xi, t) d \xi .
\end{gathered}
$$

As a result of (12)

$$
\begin{aligned}
& \left(u+u_{x}\right)(x, t)=e^{x} \int_{x}^{\infty} e^{-\xi} y(\xi, t) d \xi \\
& \left(u-u_{x}\right)(x, t)=e^{-x} \int_{-\infty}^{x} e^{\xi} y(\xi, t) d \xi
\end{aligned}
$$

for all $t \geq 0$.

From (12) and differentiating $u u_{x}\left(q\left(x_{0}, t\right), t\right)$ with respect to $t$, we have

$$
\begin{aligned}
\frac{d}{d t}\left(2 u u_{x}\right)\left(q\left(x_{0}, t\right), t\right) & \\
=\frac{d}{d t}\{- & \frac{1}{2} e^{-2 q\left(x_{0}, t\right)}\left(\int_{-\infty}^{q\left(x_{0}, t\right)} e^{\xi} y(\xi, t) d \xi\right)^{2} \\
& \left.+\frac{1}{2} e^{2 q\left(x_{0}, t\right)}\left(\int_{q\left(x_{0}, t\right)}^{\infty} e^{-\xi} y(\xi, t) d \xi\right)^{2}\right\}
\end{aligned}
$$




$$
\begin{aligned}
= & b u^{2}\left(q\left(x_{0}, t\right), t\right) e^{-2 q\left(x_{0}, t\right)}\left(\int_{-\infty}^{q\left(x_{0}, t\right)} e^{\xi} y(\xi, t) d \xi\right)^{2} \\
& -e^{-2 q\left(x_{0}, t\right)} \int_{-\infty}^{q\left(x_{0}, t\right)} e^{\xi} y(\xi, t) d \xi \int_{-\infty}^{q\left(x_{0}, t\right)} e^{\xi} y_{t}(\xi, t) d \xi \\
& +b u^{2}\left(q\left(x_{0}, t\right), t\right) e^{2 q\left(x_{0}, t\right)}\left(\int_{q\left(x_{0}, t\right)}^{\infty} e^{-\xi} y(\xi, t) d \xi\right)^{2} \\
& +e^{2 q\left(x_{0}, t\right)} \int_{q\left(x_{0}, t\right)}^{\infty} e^{-\xi} y(\xi, t) d \xi \int_{q\left(x_{0}, t\right)}^{\infty} e^{-\xi} y_{t}(\xi, t) d \xi \\
= & b u^{2}\left(u-u_{x}\right)^{2}\left(q\left(x_{0}, t\right), t\right) \\
& -\left(u-u_{x}\right)\left(q\left(x_{0}, t\right), t\right) e^{-q\left(x_{0}, t\right)} \int_{-\infty}^{q\left(x_{0}, t\right)} e^{\xi} y_{t}(\xi, t) d \xi \\
& +b u^{2}\left(u+u_{x}\right)^{2}\left(q\left(x_{0}, t\right), t\right) \\
& +\left(u+u_{x}\right)\left(q\left(x_{0}, t\right), t\right) e^{q\left(x_{0}, t\right)} \int_{q\left(x_{0}, t\right)}^{\infty} e^{-\xi} y_{t}(\xi, t) d \xi .
\end{aligned}
$$

Equation (1) can be rewritten as

$$
y_{t}=-b\left(y u^{2}\right)_{x}+(2 b-a) y u u_{x} .
$$

Firstly, we can estimate the first term as

$$
\begin{aligned}
& e^{-q\left(x_{0}, t\right)} \int_{-\infty}^{q\left(x_{0}, t\right)} e^{\xi} y_{t}(\xi, t) d \xi \\
& =-b e^{-q\left(x_{0}, t\right)} \int_{-\infty}^{q\left(x_{0}, t\right)} e^{\xi}\left(y(\xi, t) u^{2}(\xi, t)\right)_{\xi} d \xi \\
& +(2 b-a) e^{-q\left(x_{0}, t\right)} \int_{-\infty}^{q\left(x_{0}, t\right)} e^{\xi} y(\xi, t) u(\xi, t) u_{\xi}(\xi, t) d \xi \\
& =b e^{-q\left(x_{0}, t\right)} \int_{-\infty}^{q\left(x_{0}, t\right)} e^{\xi} y(\xi, t) u^{2}(\xi, t) d \xi \\
& +(2 b-a) e^{-q\left(x_{0}, t\right)} \int_{-\infty}^{q\left(x_{0}, t\right)} e^{\xi} y(\xi, t) u(\xi, t) u_{\xi}(\xi, t) d \xi \\
& =b e^{-q\left(x_{0}, t\right)} \int_{-\infty}^{q\left(x_{0}, t\right)} e^{\xi}\left(u^{3}-u^{2} u_{x x}\right) d \xi \\
& +(2 b-a) e^{-q\left(x_{0}, t\right)} \int_{-\infty}^{q\left(x_{0}, t\right)} e^{\xi}\left(u^{2} u_{x}-u u_{x} u_{x x}\right) d \xi \\
& =\frac{3 b-a}{3} u^{3}-b u^{2} u_{x}-\frac{2 b-a}{2} u u_{x}^{2} \\
& +\frac{a}{3} e^{-q\left(x_{0}, t\right)} \int_{-\infty}^{q\left(x_{0}, t\right)} e^{\xi} u^{3} d \xi
\end{aligned}
$$

$$
\begin{aligned}
& +\frac{6 b-a}{2} e^{-q\left(x_{0}, t\right)} \int_{-\infty}^{q\left(x_{0}, t\right)} e^{\xi} u u_{x}^{2} d \xi \\
& +\frac{2 b-a}{2} e^{-q\left(x_{0}, t\right)} \int_{-\infty}^{q\left(x_{0}, t\right)} e^{\xi} u_{x}^{3} d \xi
\end{aligned}
$$

We also apply the following inequality in [6]:

$$
\begin{gathered}
\int_{-\infty}^{q\left(x_{0}, t\right)} e^{\xi}\left(2 u^{3}+3 u u_{\xi}^{2}-u_{\xi}^{3}\right)(\xi, t) d \xi \\
\geq e^{q\left(x_{0}, t\right)} u^{3}\left(q\left(x_{0}, t\right), t\right) .
\end{gathered}
$$

So we can derive

$$
\begin{aligned}
& \frac{a}{3} e^{-q\left(x_{0}, t\right)} \int_{-\infty}^{q\left(x_{0}, t\right)} e^{\xi} u^{3} d \xi \\
& \quad+\frac{6 b-a}{2} e^{-q\left(x_{0}, t\right)} \int_{-\infty}^{q\left(x_{0}, t\right)} e^{\xi} u u_{x}^{2} d \xi \\
& \quad+\frac{2 b-a}{2} e^{-q\left(x_{0}, t\right)} \int_{-\infty}^{q\left(x_{0}, t\right)} e^{\xi} u_{x}^{3} d \xi \\
& =\frac{1}{6} e^{-q\left(x_{0}, t\right)} \int_{-\infty}^{q\left(x_{0}, t\right)} e^{\xi}\left[(a-3 b)\left(2 u^{3}-3 u u_{x}^{2}-3 u_{x}^{3}\right)\right. \\
& \geq \frac{a-3 b}{6} u^{3}+\frac{3 b}{6} u^{3}=\frac{a}{6} u^{3} .
\end{aligned}
$$

Putting (19) into (17), we have

$$
\begin{aligned}
& e^{-q\left(x_{0}, t\right)} \int_{-\infty}^{q\left(x_{0}, t\right)} e^{\xi} y_{t}(\xi, t) d \xi \\
& \geq-b u^{2}\left(q\left(x_{0}, t\right), t\right) u_{x}\left(q\left(x_{0}, t\right), t\right) \\
& \quad-\frac{2 b-a}{2} u\left(q\left(x_{0}, t\right), t\right) u_{x}^{2}\left(q\left(x_{0}, t\right), t\right) \\
& +\frac{6 b-a}{6} u^{3}\left(q\left(x_{0}, t\right), t\right) .
\end{aligned}
$$

Similarly, we have

$$
\begin{aligned}
& e^{q\left(x_{0}, t\right)} \int_{q\left(x_{0}, t\right)}^{\infty} e^{-\xi} y_{t}(\xi, t) d \xi \\
& \geq-b u^{2}\left(q\left(x_{0}, t\right), t\right) u_{x}\left(q\left(x_{0}, t\right), t\right) \\
& +\frac{2 b-a}{2} u\left(q\left(x_{0}, t\right), t\right) u_{x}^{2}\left(q\left(x_{0}, t\right), t\right) \\
& +\frac{a-6 b}{6} u^{3}\left(q\left(x_{0}, t\right), t\right) .
\end{aligned}
$$


Putting (21) and (22) into (15), we obtain

$$
\begin{aligned}
\frac{d}{d t} 2 u u_{x}\left(q\left(x_{0}, t\right), t\right) & \\
\leq & 2 b\left(u^{4}+u^{2} u_{x}^{2}\right)\left(q\left(x_{0}, t\right), t\right) \\
& -\left(u-u_{x}\right)\left(\frac{6 b-a}{6} u^{3}-b u^{2} u_{x}-\frac{2 b-a}{2} u u_{x}^{2}\right) \\
& +\left(u+u_{x}\right)\left(-\frac{6 b-a}{6} u^{3}-b u^{2} u_{x}+\frac{2 b-a}{2} u u_{x}^{2}\right) \\
= & \frac{a}{3} u^{2}\left(u^{2}-u_{x}^{2}\right)\left(q\left(x_{0}, t\right), t\right) .
\end{aligned}
$$

Here we use the facts that $\left(u-u_{x}\right)(q(x, t), t) \geq 0, x \in$ $\left(-\infty, q\left(x_{0}, t\right)\right)$, from (10) and (14), and $\left(u+u_{x}\right)(q(x, t), t) \leq 0$, $x \in\left(q\left(x_{0}, t\right), \infty\right)$, from (10) and (13).

Claim 1. $u u_{x}\left(q\left(x_{0}, t\right), t\right)<0$ is decreasing and $u^{2}\left(q\left(x_{0}, t\right), t\right)<$ $u_{x}^{2}\left(q\left(x_{0}, t\right), t\right)$ for all $t \geq 0$.

Suppose that there exists a $t_{0}$ such that $u^{2}\left(q\left(x_{0}, t\right), t\right)<$ $u_{x}^{2}\left(q\left(x_{0}, t\right), t\right)$ on $\left[0, t_{0}\right)$ and $u^{2}\left(q\left(x_{0}, t_{0}\right), t_{0}\right) \quad \geq$ $u_{x}^{2}\left(q\left(x_{0}, t_{0}\right), t_{0}\right)$. Now, let

$$
\begin{aligned}
& I(t):=u\left(u-u_{x}\right)\left(q\left(x_{0}, t\right), t\right), \\
& I I(t):=u\left(u+u_{x}\right)\left(q\left(x_{0}, t\right), t\right) .
\end{aligned}
$$

Firstly, differentiating $I(t)$, we get

$$
\begin{aligned}
& \frac{d I(t)}{d t}=-b u^{2}\left(q\left(x_{0}, t\right), t\right) \\
& \times\left(e^{-q\left(x_{0}, t\right)} \int_{-\infty}^{q\left(x_{0}, t\right)} e^{\xi} y(\xi, t) d \xi\right)^{2} \\
& +\left(e^{-q\left(x_{0}, t\right)} \int_{-\infty}^{q\left(x_{0}, t\right)} e^{\xi} y(\xi, t) d \xi\right) \\
& \times\left(e^{-q\left(x_{0}, t\right)} \int_{-\infty}^{q\left(x_{0}, t\right)} e^{\xi} y_{t}(\xi, t) d \xi\right) \\
& +\frac{1}{2} \int_{-\infty}^{q\left(x_{0}, t\right)} e^{\xi} y_{t}(\xi, t) d \xi \int_{q\left(x_{0}, t\right)}^{\infty} e^{-\xi} y(\xi, t) d \xi \\
& +\frac{1}{2} \int_{-\infty}^{q\left(x_{0}, t\right)} e^{\xi} y(\xi, t) d \xi \int_{q\left(x_{0}, t\right)}^{\infty} e^{-\xi} y_{t}(\xi, t) d \xi \\
& \geq-b u^{2}\left(u-u_{x}\right)^{2}\left(q\left(x_{0}, t\right), t\right) \\
& +\left(\frac{6 b-a}{6} u^{3}-b u^{2} u_{x}-\frac{2 b-a}{2} u u_{x}^{2}\right) u\left(q\left(x_{0}, t\right), t\right) \\
& +\frac{1}{2}\left(\frac{6 b-a}{6} u^{3}-b u^{2} u_{x}-\frac{2 b-a}{2} u u_{x}^{2}\right) \\
& \times\left(u-u_{x}\right)\left(q\left(x_{0}, t\right), t\right)
\end{aligned}
$$

$$
\begin{aligned}
& -\frac{1}{2}\left(\frac{6 b-a}{6} u^{3}+b u^{2} u_{x}-\frac{2 b-a}{2} u u_{x}^{2}\right) \\
& \times\left(u-u_{x}\right)\left(q\left(x_{0}, t\right), t\right) \\
\geq & \frac{a}{6} u^{2}\left(u_{x}^{2}-u^{2}\right), \quad \text { on }\left[0, t_{0}\right) .
\end{aligned}
$$

Secondly, differentiating $I I(t)$, we get

$$
\begin{aligned}
& \frac{d I I(t)}{d t}=b u^{2}\left(q\left(x_{0}, t\right), t\right) \\
& \times\left(e^{q\left(x_{0}, t\right)} \int_{q\left(x_{0}, t\right)}^{\infty} e^{-\xi} y(\xi, t) d \xi\right)^{2} \\
& +\left(e^{q\left(x_{0}, t\right)} \int_{q\left(x_{0}, t\right)}^{\infty} e^{-\xi} y(\xi, t) d \xi\right) \\
& \times\left(e^{q\left(x_{0}, t\right)} \int_{q\left(x_{0}, t\right)}^{\infty} e^{-\xi} y_{t}(\xi, t) d \xi\right) \\
& +\frac{1}{2} \int_{-\infty}^{q\left(x_{0}, t\right)} e^{\xi} y_{t}(\xi, t) d \xi \int_{q\left(x_{0}, t\right)}^{\infty} e^{-\xi} y(\xi, t) d \xi \\
& +\frac{1}{2} \int_{-\infty}^{q\left(x_{0}, t\right)} e^{\xi} y(\xi, t) d \xi \int_{q\left(x_{0}, t\right)}^{\infty} e^{-\xi} y_{t}(\xi, t) d \xi \\
& \leq b u^{2}\left(u+u_{x}\right)^{2}\left(q\left(x_{0}, t\right), t\right) \\
& -\left(\frac{6 b-a}{6} u^{3}+b u^{2} u_{x}-\frac{2 b-a}{2} u u_{x}^{2}\right) \\
& \times u_{x}\left(q\left(x_{0}, t\right), t\right) \\
& +\frac{1}{2}\left(\frac{6 b-a}{6} u^{3}-b u^{2} u_{x}-\frac{2 b-a}{2} u u_{x}^{2}\right) \\
& \times\left(u+u_{x}\right)\left(q\left(x_{0}, t\right), t\right) \\
& -\frac{1}{2}\left(\frac{6 b-a}{6} u^{3}+b u^{2} u_{x}-\frac{2 b-a}{2} u u_{x}^{2}\right) \\
& \times\left(u+u_{x}\right)\left(q\left(x_{0}, t\right), t\right) \\
& =-\frac{a}{6} u^{2}\left(u_{x}^{2}-u^{2}\right) \text {, on }\left[0, t_{0}\right) \text {. }
\end{aligned}
$$

Hence, from (24), (25), and the continuity property of ODEs, we can draw

$$
u^{2}\left(u_{x}^{2}-u^{2}\right)\left(q\left(x_{0}, t\right), t\right)=-I(t) I I(t)>-I(0) I I(0)>0,
$$

for all $t>0$. This means $t_{0}$ can be extended to infinity. This is a contradiction, so the claim is true. 
Moreover, using (24) and (25) again, we have the following inequality for $u^{2}\left(u_{x}^{2}-u^{2}\right)\left(q\left(x_{0}, t\right), t\right)$ :

$$
\begin{aligned}
& \frac{d}{d t} u^{2}\left(u_{x}^{2}-u^{2}\right)\left(q\left(x_{0}, t\right), t\right) \\
& \quad \geq-\frac{a}{3} u u_{x}\left(q\left(x_{0}, t\right), t\right) u^{2}\left(u_{x}^{2}-u^{2}\right)\left(q\left(x_{0}, t\right), t\right)
\end{aligned}
$$

Putting (22) into (27) yields

$$
\begin{aligned}
& \frac{d}{d t} u^{2}\left(u_{x}^{2}-u^{2}\right)\left(q\left(x_{0}, t\right), t\right) \\
& \geq \frac{a}{3} u^{2}\left(u_{x}^{2}-u^{2}\right)\left(q\left(x_{0}, t\right), t\right) \\
& \quad \times\left(\frac{a}{6} \int_{0}^{t} u^{2}\left(u_{x}^{2}-u^{2}\right)\left(q\left(x_{0}, s\right), s\right) d s-\frac{a}{6} u_{0} u_{0 x}\left(x_{0}\right)\right)
\end{aligned}
$$

Before completing the proof, we want the following technical lemma.

Lemma 5 (see [15]). Suppose that $\Phi$ is a twice continuous differential satisfying

$$
\begin{gathered}
\Phi^{\prime \prime}(t) \geq C_{0} \Phi^{\prime}(t) \Phi(t), \quad t>0, C_{0}>0 \\
\Phi(0)>0, \quad \Phi^{\prime}(0)>0
\end{gathered}
$$

Then $\Phi(t)$ blows up in finite time. Moreover the blow-up time $T$ can be estimated in terms of the initial datum as

$$
T \leq \max \left\{\frac{2}{C_{0} \Phi(0)}, \frac{\Phi(0)}{\Phi^{\prime}(0)}\right\} .
$$

Let $\Phi(t)=\int_{0}^{t} u^{2}\left(u_{x}^{2}-u^{2}\right)\left(q\left(x_{0}, s\right), s\right) d s-u_{0} u_{0 x}\left(x_{0}\right)$; then (28) is an equation of type (29) with $C_{0}=a^{2} / 18$. The proof is complete by applying Lemma 5 .

When we change the signs of $u_{0}\left(x_{0}\right)$ and $y_{0}(x)$ in Theorem 4 , similarly, we have the following blow-up criterion.

Theorem 6. If $a>3 b$, suppose that $u_{0} \in H^{s}(\mathbb{R}), s>(3 / 2)$, and there exists a $x_{0} \in \mathbb{R}$ such that $u_{0}\left(x_{0}\right) \leq 0$ and $y_{0}\left(x_{0}\right)=$ $\left(1-\partial_{x}^{2}\right) u_{0}\left(x_{0}\right)=0$,

$$
\begin{gathered}
y_{0}(x) \leq 0(\neq \equiv 0) \quad \text { for } x \in\left(-\infty, x_{0}\right), \\
y_{0}(x) \geq 0(\not \equiv 0) \quad \text { for } x \in\left(x_{0}, \infty\right) .
\end{gathered}
$$

Then the corresponding solution $u(x, t)$ to the modified Novikov equation (1) with $u_{0}(x)$ as the initial datum blows up in finite time.

\section{Conflict of Interests}

The authors declare that there is no conflict of interests regarding the publication of this paper.

\section{Acknowledgment}

The authors thank Doctor Zaihong Jiang for patient guidance and helpful discussion.

\section{References}

[1] S. Lai, H. Yan, and N. Li, "The global solution and blow-up phenomena to a modified Novikov equation," Boundary Value Problems, vol. 2014, no. 1, p. 16, 2014.

[2] V. Novikov, "Generalizations of the Camassa-Holm equation," Journal of Physics A: Mathematical and Theoretical, vol. 42, no. 34, Article ID 342002, 2009.

[3] A. N. W. Hone, H. Lundmark, and J. Szmigielski, "Explicit multipeakon solutions of Novikov's cubically nonlinear integrable Camassa-Holm type equation," Dynamics of Partial Differential Equations, vol. 6, no. 3, pp. 253-289, 2009.

[4] A. N. W. Hone and J. P. Wang, "Integrable peakon equations with cubic nonlinearity," Journal of Physics A: Mathematical and Theoretical, vol. 41, no. 37, Article ID 372002, 2008.

[5] L. Ni and Y. Zhou, "Well-posedness and persistence properties for the Novikov equation," Journal of Differential Equations, vol. 250, no. 7, pp. 3002-3021, 2011.

[6] Z. Jiang and L. Ni, "Blow-up phenomenon for the integrable novikov equation," Journal of Mathematical Analysis and Applications, vol. 385, no. 1, pp. 551-558, 2012.

[7] C. Ma and Y. Jin, "Blow-up of the weakly dissipative Novikov equation," Journal of Inequalities and Applications, vol. 2014, no. 1, p. 9, 2014.

[8] S. Zhou and R. Chen, "A few remarks on the generalized Novikov equation," Journal of Inequalities and Applications, vol. 2013, no. 1, p. 560, 2013.

[9] X. Wu and Z. Yin, "Global weak solutions for the Novikov equation," Journal of Physics A: Mathematical and Theoretical, vol. 44, no. 5, Article ID 055202, 2011.

[10] W. Yan, Y. Li, and Y. Zhang, "The Cauchy problem for the integrable Novikov equation," Journal of Differential Equations, vol. 253, no. 1, pp. 298-318, 2012.

[11] L. Zhao and S. Zhou, "Symbolic analysis and exact travelling wave solutions to a new modified Novikov equation," Applied Mathematics and Computation, vol. 217, no. 2, pp. 590-598, 2010.

[12] A. A. Himonas and C. Holliman, "The Cauchy problem for the Novikov equation,” Nonlinearity, vol. 25, no. 2, pp. 449-479, 2012.

[13] S. Lai and M. Wu, "The local strong and weak solutions to a generalized Novikov equation," Boundary Value Problems, vol. 2013, no. 1, pp. 1-12, 2013.

[14] Y. Zhou, "Wave breaking for a shallow water equation," Nonlinear Analysis, Theory, Methods and Applications, vol. 57, no. 1, pp. 137-152, 2004.

[15] Y. Zhou, "On solutions to the Holm-Staley b-family of equations," Nonlinearity, vol. 23, no. 2, pp. 369-381, 2010. 


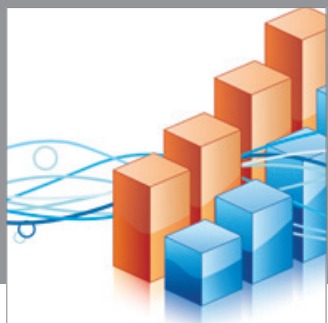

Advances in

Operations Research

mansans

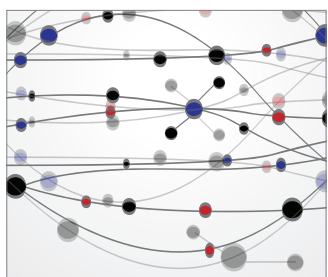

The Scientific World Journal
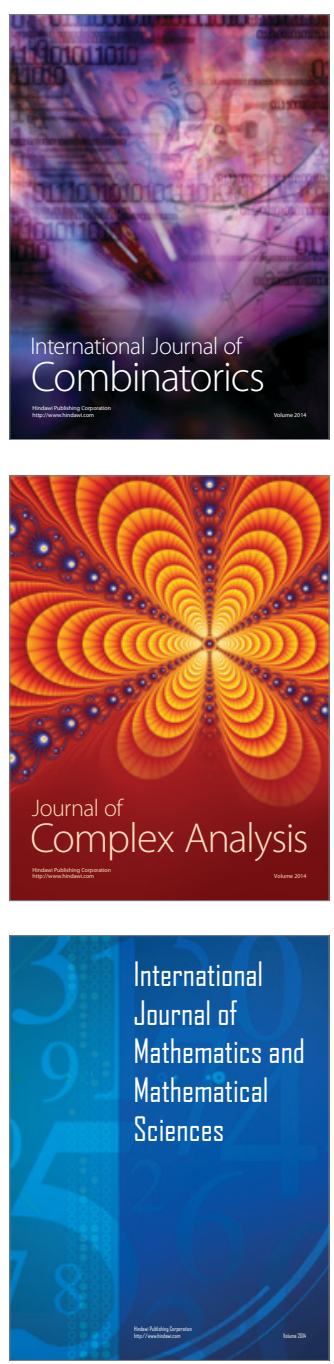
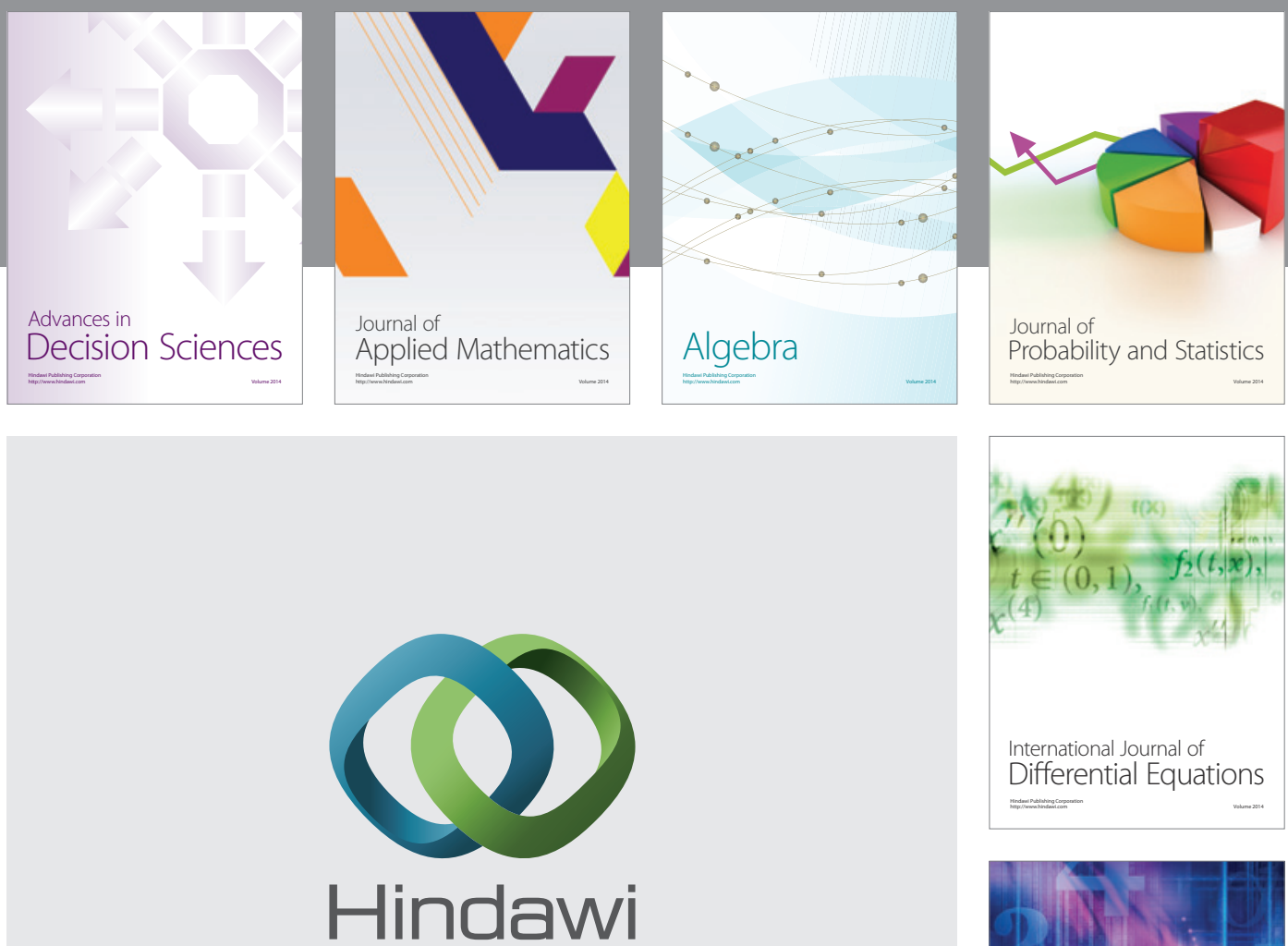

Submit your manuscripts at http://www.hindawi.com
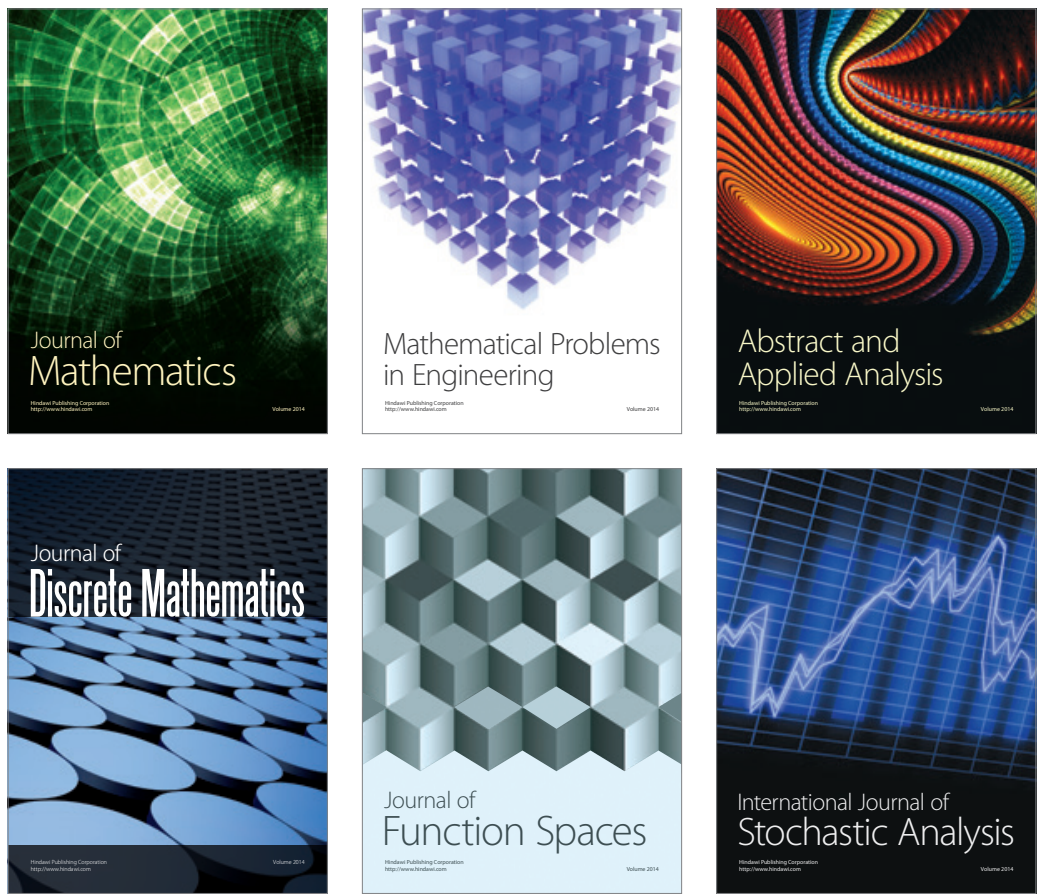

Journal of

Function Spaces

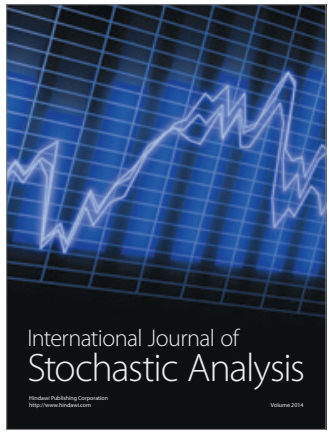

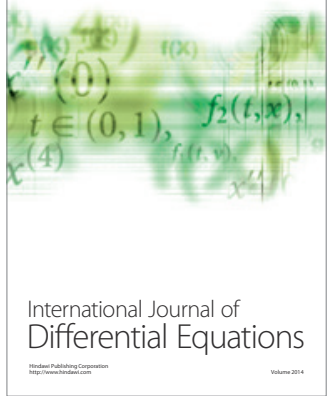
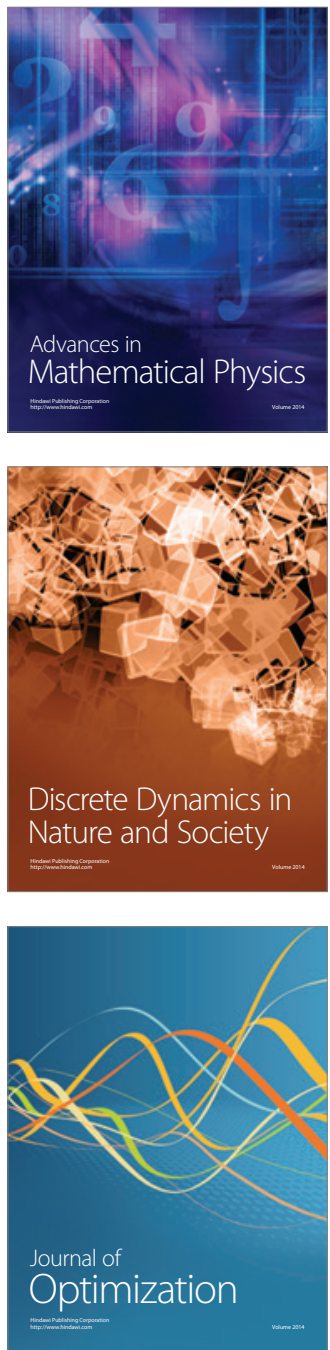